\title{
Effect of Predictive Nursing Intervention in Preventing Complicated Phlebitis in Colon Cancer Patients Receiving Peripherally Inserted Central Catheter
}

\author{
Y. Y. HE, Y. H. LI ${ }^{1 *}$, F. TENG ${ }^{1}$, S. QIE 1 , W. S. ZHOU 2 , X. H. LIU ${ }^{1}$, J. QI ${ }^{3}$ AND H. Y. SHI ${ }^{1}$
}

Department of Medical Oncology, Baoding No.1 Central Hospital, ${ }^{1}$ Department of Radiation Therapy, Affiliated Hospital of Heibei University, ${ }^{2}$ Department of General Surgery, Baoding No.1 Central Hospital, ${ }^{3}$ Department of Galactophore Surgery, Affiliated Hospital of Heibei University, Baoding 071000, China

\section{He et al.: Predictive Nursing Intervention in Preventing Complicated Phlebitis}

To observe the effect of predictive nursing intervention in preventing complicated phlebitis in colon cancer patients receiving peripherally inserted central catheter. A total of 115 colon cancer patients who underwent peripherally inserted central catheter in our hospital from June 2018 to June 2020 were selected as the study subjects and divided into 2 groups according to the order of admission. 57 cases in the control group received routine nursing intervention. 58 patients in the study group received predictive nursing intervention. The occurrence of phlebitis, the first occurrence time of phlebitis, the average catheterization duration and nursing satisfaction were compared between the two groups. The total incidence of phlebitis was $6.90 \%$ in the study group, which was lower than $21.05 \%$ of the control group, showing statistically significant difference between the groups $(p<0.05)$. The study group had later first occurrence time of phlebitis $(\mathbf{p}<\mathbf{0 . 0 5})$ and longer average catheterization duration than the control group $(p<0.05)$. The nursing satisfaction was $91.38 \%$ in the study group, which was higher than $77.19 \%$ of the control group, showing statistically significant difference between the groups $(\mathbf{p}<0.05)$. Predictive nursing intervention can effectively prevent complicated phlebitis in colon cancer patients receiving peripherally inserted central catheter, delay the occurrence of phlebitis, prolong the average catheterization duration and receive high patient satisfaction.

Key words: Predictive nursing, colon cancer, peripherally inserted central catheter, phlebitis, preventive effect

Colon cancer is a high incidence disease in our country, whose morbidity and mortality are on the rise according to survey ${ }^{[1]}$. Chemotherapy is an important method for clinical treatment of colon cancer. Due to the long chemotherapy cycle, chemotherapy drugs will inevitably stimulate blood vessels. Thanks to rapid advancement in venipuncture tools and techniques, venipuncture may provide effective means to protect blood vessels and guarantee timely and effective infusion of chemotherapy drugs. In particular, peripherally inserted central catheter (PICC) technology is widely used in clinics due to its simple operation and high safety factor. By establishing a central venous access, it can avoid vascular damage and local irritation on the premise of smooth progress of the chemotherapy regimen, thus suitable for colon cancer chemotherapy ${ }^{[2]}$.
However, the application of PICC for chemotherapy is prone to a variety of complications, the most common of this is phlebitis ${ }^{[3]}$. Occurrence of phlebitis significantly increases the patient's subjective pain perception, prolongs the hospital stay and even reduces the efficacy of radiotherapy and chemotherapy. Studies have pointed out that application of PICC for radiotherapy and chemotherapy requires regular maintenance and appropriate nursing intervention ${ }^{[4]}$. Predictive nursing intervention is to make comprehensive judgments based on past experience, medical knowledge and patient reality, clarify the existing or potential problems in the patient, provide targeted prevention and treatment measures and efficiently deal with the existing or potential problems in the patient ${ }^{[5]}$. Based on this, this study adopts predictive nursing intervention to prevent

*Address for correspondence

E-mail: liyanhong2020@126.com 
complicated phlebitis in colon cancer patients receiving PICC. The intervention effect is reported as follows. 115 colon cancer patients who underwent PICC in our hospital from June 2018 to June 2020 were selected as the study subjects and divided into 2 groups according to the order of admission. There were 57 cases in the control group, including 40 males, 17 females, aged 45 69 y old, the patients had an average age of (61.64 \pm 3.59$) \mathrm{y}$, weighing 55 84 kg, the patients had an average weight of $(70.86 \pm 6.47) \mathrm{kg}$. There were 58 cases in the study group, including 42 males and 16 females, aged $45 \sim 70 \mathrm{y}$ old, the patients had an average age of (61.72 \pm 3.64$) \mathrm{y}$, weighing $56 \sim 85 \mathrm{~kg}$, the patients had an average weight of $(70.93 \pm 6.52) \mathrm{kg}$. There was no statistically significant difference in general information between the two groups ( $>0.05$ ), showing comparability. This study was reviewed and approved by the hospital ethics committee. Inclusion criteria: Meet the diagnostic criteria for colon cancer $^{[6]}$ and confirmed by pathological examination; meet PICC catheterization indications; had no venous thrombosis disease previously, have normal communication and understanding skills; patients are informed, agree to participate in this study. Exclusion criteria: Severe heart, liver and kidney dysfunction; (previously) suffering from mental illness; having other malignant tumors; having blood system diseases, or in allergic constitution; lack of clinical data. Control group: Routine nursing intervention was given, including the following aspects: Implement nursing intervention in accordance with doctor's advice and routine nursing requirements. The arm circumference was measured before PICC catheterization. On the day of PICC catheterization, wet and hot compresses were applied for $0.5 \mathrm{~h}, 3$ times/d from the next day for $4 \mathrm{~d}$; on the day of PICC catheterization, clench fist, $10 \mathrm{~min} /$ time, 4 times/d and raise the affected limb. Observe the puncture point every day to see if there are any abnormalities such as blood oozing and fluid oozing. Study group: Predictive nursing intervention was given for 3 mo. A predictive nursing intervention team was established. Team members include the attending physician, associate chief nurse, chief nurse and nurse. All staff members were trained before joining the group, who should learn about the content of predictive nursing intervention and related theoretical knowledge of phlebitis. "PICC-induced Phlebitis Prevention Guide" was drafted and distributed to each patient. Based on responsibility system of nursing, clarify responsible nurse to each patient. The responsible nurse makes preliminary judgments based on the basic information and specific conditions of each patient, finds out the defects in the nursing intervention process and proposes prevention plan based on the previous clinical nursing experience and medical nursing knowledge. The plan includes pretreatment before PICC catheterization: Before catheterization, soak the PICC catheter with dexamethasone and reasonably combine $5 \mathrm{mg}$ dexamethasone $+50 \mathrm{ml} 0.9 \%$ sodium chloride injection. Selection of puncture vein: patient has higher comfort when blood vessel lumen is large, path is straight and fast blood flow. The recommended puncture veins are successively basilic vein, median cubital vein, cephalic vein and brachial vein. Timing of catheterization: PICC catheterization should be performed $2 \mathrm{~d}$ before chemotherapy. Correct catheterization position: take the supine position during catheterization, abduct the upper extremity 90 on the catheterization side, tilt the head to the catheterization side and press the lower jaw against the shoulder. Catheter fixation: The upper elbow vein puncture is " $U$ "-shape fixed and the lower elbow vein puncture is " $\mathrm{C}$ "-shape fixed. Once the catheter is folded due to limb activity, the catheter needs to be repositioned, adjusted and fixed, or a catheter fixer can be used. Positive pressure joint and transparent applicator replacement: The responsible nurse replaces the positive pressure joint and transparent applicator for the patient once a week. It is necessary to strictly standardize the catheterization operation. For example, pay attention to gentle movements and precise techniques during the catheterization process, strictly implement aseptic operations and perform regular disinfection. Do not continue to deliver the catheter when venous spasm occurs. During interruption of the delivery, regular injection of sodium chloride is required. Correctly and effectively perform pipe sealing, flushing, etc. Health education: adopt appropriate publicity and education methods in light of each patient's academic qualifications, work, etc. The publicity and education content transits from shallow to deep one, introducing colon cancer, PICC catheterization (purpose, process, precautions, etc.), phlebitis, etc., in language simple and easy to understand. Do a good job in catheter protection, wrap it with an appropriate amount of plastic before bathing and wipe it locally with a towel in time after bathing to effectively identify abnormalities. Do not continue to compress the catheterization side in daily activities; learn some rehabilitation exercises for the catheterization side limb, including finger exercises, fisting exercises, wrist rotation exercises, etc. Nursing intervention during chemotherapy: the staff should know the $\mathrm{pH}$ value, 
effect and safe dilution of the chemotherapy regimen and can accurately arrange the order of drug use. First give less irritating drugs, then give more irritating drugs and similar irritating drugs should be used with an interval $>20$ min. Mix Shengmai injection $25 \mathrm{ml}+5 \%$ glucose injection $250 \mathrm{ml}$ for intravenous instillation once before and after chemotherapy. Comparison of occurrence of phlebitis is mainly based on the phlebitis severity assessment standard of the intravenous nurses society $(\mathrm{INS})^{[7]}$, of which, grade 0 : No clinical symptoms; grade 1: Redness or pain at the catheterization site; grade 2: Pain at the catheterization site, accompanied by redness, swelling, etc.; grade 3: Pain at the catheterization site, accompanied by redness, swelling, etc., and cord-like veins are touchable; grade 4: Pain at the catheterization site, accompanied by redness, swelling, etc., cord-like veins are touchable (more than $2.54 \mathrm{~cm}$ in length), accompanied by outflow of pus. Total incidence of phlebitis in each group was counted, that is, the proportion of grade $1+$ proportion of grade 2+proportion of grade 3+proportion of grade 4. Comparison of the first occurrence time of phlebitis and the average catheterization duration; the first occurrence time of phlebitis in each group was recorded and the average catheterization duration of each group was calculated. Comparison of nursing satisfaction; Satisfaction survey questionnaire was self-made, with survey results divided into very satisfied, generally satisfied and dissatisfied and the satisfaction of each group was calculated, that is, proportion of very satisfied + generally satisfied patients. The data obtained in this study are all analyzed using statistical software SPSS 22.0. Measurement data conforming to normal distribution are indicated by mean \pm standard deviation and independent sample $t$ test is used for comparison between groups; counting data is indicated by percentage and $\chi^{2}$ test is taken for comparison between groups. The test level $\alpha=0.05, \quad \mathrm{p}<0.05$ indicates statistically significant difference. The total incidence of phlebitis is $6.90 \%$ in the study group, which is lower than $21.05 \%$ of the control group, showing statistically significant difference between the groups $(p<0.05)$, as shown in Table 1. The study group has later first occurrence time of phlebitis $(\mathrm{p}<0.05)$ and longer average catheterization duration than the control group $(\mathrm{p}<0.05)$, as shown in Table 2. The nursing satisfaction is $91.38 \%$ in the study group, which is higher than $77.19 \%$ of the control group, showing statistically significant difference between the groups $(p<0.05)$, as shown in Table $3(\mathrm{p}<0.05)$. Colon cancer needs relatively long chemotherapy cycle ${ }^{[8,9]}$. Patients need review blood routines regularly at hospital following the doctor's instructions to prepare for the formulation/ adjustment of the next chemotherapy regimen. Most patients elbow veins are repeatedly punctured, so chemotherapy drugs will damage and stimulate the blood vessels, while puncture tools and techniques eliminate the need for repeated punctures, so the established effective venous access can better protect the blood vessels ${ }^{[10,11]}$. At present, PICC technology is often adopted in tumor radiotherapy and chemotherapy due to its safety, convenience and comfort ${ }^{[12]}$. Investigations show that phlebitis is a common complication after PICC catheterization, with an incidence rate as high as $16 \%$. It mostly occurs within 3 d after PICC catheterization ${ }^{[13]}$. Without timely symptomatic treatment, not only the expected PICC catheterization purpose will not be achieved, but also the treatment efficiency and patients quality of life will be reduced, bringing great pain to patients both physically and mentally ${ }^{[14]}$. Studies have pointed out that the occurrence of phlebitis after PICC catheterization involves a variety of factors, including vein puncture in PICC and patient psychological factors ${ }^{[15]}$. Therefore, it is necessary to actively take effective measures to prevent phlebitis in PICC application and prevention is the key. Zhang et al. ${ }^{[16]}$ reported that supplemented scientific and appropriate nursing interventions can significantly reduce the occurrence of phlebitis after PICC catheterization, which plays an exact preventive role, provides favorable conditions for PICC indwelling and extends PICC indwelling time. Predictive nursing intervention characterized by foresight and pertinence is to make comprehensive judgments based on past experience, medical knowledge and patient reality to clarify the existing or potential problems in the patient. Based on this, it supplements targeted prevention and treatment measures to efficiently handle the existing or potential problems in the patient ${ }^{[17]}$. In this study, predictive nursing intervention is used to prevent phlebitis in colon cancer patients receiving PICC. Nursing staff formulate comprehensive and scientific targeted intervention programs based on past experience, medical knowledge and patient reality to promote the initiative and enthusiasm of colon cancer patients, increase PICC tolerance, thereby preventing the occurrence of phlebitis after PICC catheterization. The key to the implementation of predictive nursing intervention is to familiarize with the high-risk factors 
of phlebitis, adhere to the principle of prevention first, so that nursing staff can effectively implement various nursing measures in a targeted and efficient manner ${ }^{[18]}$. In this study, predictive nursing intervention was used to prevent complicated phlebitis in colon cancer patients receiving PICC. Conforming to the principle of "prevention first", nursing staff proceed from multiple aspects of PICC catheterization pretreatment, choice of puncture vein, catheterization duration, health education, etc. accurately identify high-risk factors of complicated phlebitis in PICC, make objective evaluation and effective control with clear focus in nursing intervention. The results showed that the total incidence of phlebitis was $6.90 \%$ in the study group, which was lower than $21.05 \%$ of the control group, showing significant difference between the groups, indicating that predictive nursing intervention can effectively prevent complicated phlebitis in colon cancer patients receiving PICC. The analysis believes that the implementation of predictive nursing based on the patient reality, the phlebitis generation mechanism and progress law, etc. can effectively identify and predict the occurrence of phlebitis in advance. The various measures in the nursing intervention plan are more targeted. Based on simultaneous implementation of multiple interventions such as superb puncture skills, appropriate timing of puncture, correct positioning, etc. it can reduce the incidence of phlebitis. The results of this study also indicate that: The study group has later first occurrence time of phlebitis and longer average catheterization duration than the control group; the nursing satisfaction is $91.38 \%$ in the study group, which is higher than $77.19 \%$ of the control group, showing statistically significant difference between the groups. It suggests that predictive nursing intervention not only effectively prevent complicated phlebitis in colon cancer patients receiving PICC, but also delay the occurrence of phlebitis, prolong the average catheterization duration and receive high patient satisfaction. The analysis believes that implementation of predictive nursing interventions is mainly based on past experience, medical knowledge, patient reality, etc. The occurrence of phlebitis has been objectively evaluated and effectively predicted. With more targeted various intervention measures, it takes into account the patients psychological and physical nursing needs, helps enhance the nurse-patient relationship, so that patients fully trust and cooperate with the nursing staff. Therefore, the nursing work can be carried out smoothly and the nursing goals can be more easily achieved. To conclude, the implementation of predictive nursing intervention can effectively prevent complicated phlebitis in colon cancer patients receiving PICC, delay the occurrence of phlebitis, prolong the average catheterization duration and receive high patient satisfaction. Based on comprehensive analysis, this study believes that the key to prevent complicated phlebitis in colon cancer patients receiving PICC should adhere to the principle of prevention first. It is necessary to accurately identify high-risk factors and actively intervene after early detection. As long as proper nursing is taken, it can also achieve the goal of protecting peripheral blood vessels, reducing phlebitis incidence and increasing the patient's physical and mental comfort.

TABLE 1: COMPARISON OF OCCURRENCE OF PHLEBITIS CASES (\%)

\begin{tabular}{lccccccc}
\hline Group & Number of cases & Grade 0 & Grade 1 & Grade 2 & Grade 3 & Grade 4 & Total incidence \\
\hline $\begin{array}{l}\text { Control } \\
\text { group }\end{array}$ & 57 & $45(78.95)$ & $5(8.77)$ & $4(7.02)$ & $2(3.51)$ & $1(1.75)$ & $12(21.05)$ \\
Study group & 58 & $54(93.10)$ & $2(3.45)$ & $1(1.72)$ & $1(1.72)$ & $0(0.00)$ & $4(6.90)$ \\
$\chi^{2}$ & - & - & - & - & - & - & 4.810 \\
$\mathrm{p}$ & - & - & - & - & - & - & 0.028 \\
\hline
\end{tabular}

TABLE 2: COMPARISON OF FIRST OCCURRENCE TIME OF PHLEBITIS AND THE AVERAGE CATHETERIZATION DURATION ( $\overline{\mathbf{x}} \pm \mathbf{s})$

\begin{tabular}{lccc}
\hline Group & Number of cases & $\begin{array}{c}\text { First occurrence time of } \\
\text { phlebitis (d) }\end{array}$ & $\begin{array}{c}\text { Average catheterization } \\
\text { duration }(\mathrm{h})\end{array}$ \\
\hline Control group & 57 & $3.54 \pm 1.16$ & $73.63 \pm 11.24$ \\
Study group & 58 & $7.12 \pm 1.89$ & $100.87 \pm 11.09$ \\
$\mathrm{t}$ & - & 12.217 & 13.082 \\
$\mathrm{p}$ & - & 0.000 & 0.000 \\
\hline 176 & Indian Journal of Pharmaceutical Sciences & Special Issue 4, 2021
\end{tabular}


TABLE 3: COMPARISON OF NURSING SATISFACTION CASE (\%)

\begin{tabular}{lccccc}
\hline Group & Number of cases & Very satisfied & Generally satisfied & Dissatisfied & Satisfaction \\
\hline Control group & 57 & $15(26.32)$ & $29(50.88)$ & $13(22.81)$ & $44(77.19)$ \\
Study group & 58 & $29(50.00)$ & $24(41.38)$ & $5(8.62)$ & $53(91.38)$ \\
$\chi^{2}$ & - & - & - & - & 4.382 \\
$\mathrm{p}$ & - & - & - & - & 0.036 \\
\hline
\end{tabular}

\section{Conflicts of interest:}

The authors declared no conflict of interest.

\section{REFERENCES}

1. Bai YM, Xue L. Study on the clinical value of neoadjuvant chemotherapy in the treatment of patients with advanced colon cancer. Shanxi Med J 2019;48(7):48-50.

2. Li Y, Li T, Wei Z. The clinical characteristics and preventive measures of PICC catheter bloodstream infection in patients with malignant tumors during radiotherapy and chemotherapy. Chin J Nosocomiology 2019;29(6):872-5.

3. Xie SJ, Jiang LH. Effects of ultrasound-guided modified sedinger puncture in reducing phlebitis and venous thrombosis in patients undergoing tumor chemotherapy after peripherally inserted central catheter. Chin J Thromb Hemost 2018;24(3):429-31.

4. $\mathrm{Hu}$ JL. Observation on the effect of comprehensive nursing intervention in patients with traumatic liver and spleen rupture. World Latest Med Inf 2017;17(29):206-8.

5. Pan YH, Xiao F. Evidence-based nursing in cancer patients, psychological nursing, the application of value evaluation. Chin J Cancer Prev Treat 2018;25(S1):283-4.

6. Bai XS, Lin GL. Interpretation of updated NCCN Guidelines for colon cancer and rectal cancer. Chin Gen Pract 2019;22(33):4031-4.

7. Cai R, Lin B. The effect of early predictive nursing intervention on mechanical phlebitis after PICC in tumor patients. Lab Med Clin 2017;14(5):706-8.

8. Zhang X, Zhao H, Shi X, Jia X, Yang Y. Identification and validation of an immune-related gene signature predictive of overall survival in colon cancer. Aging 2020;12(24):26095120.

9. Kulthanachairojana N, Chansriwong P, Thokanit NS, Sirilerttrakul S, Wannakansophon N, Taychakhoonavudh S. Home-based chemotherapy for stage III colon cancer patients in Thailand: Cost-utility and budget impact analyses. Cancer Med 2021;10(3):1027-33.
10. Andre T, Vernerey D, Mineur L, Bennouna J, Desrame J, Faroux R, et al. Three versus 6 months of oxaliplatin-based adjuvant chemotherapy for patients with stage III colon cancer: disease-free survival results from a randomized, open-label, International duration evaluation of adjuvant (IDEA) France, phase III trial. J Clin Oncol 2018;36(15):1469-77.

11. Lee H, Mansouri M, Tajmir S, Lev MH, Do S. A deep-learning system for fully-automated peripherally inserted central catheter (PICC) tip detection. J Digit Imaging 2018;31(4):393402.

12. Hou H, Jin J. Influence of delaying removal of bracing guide wire on successful rate of catheter misplacement restoration during catheterization of PICC. Mod Med Health 2014;30(21):3220-2.

13. Bae SU, Jeong WK, Baek SK. Complete mesocolic excision and central vascular ligation for locally invasive right-sided colon cancer by an open approach-a video vignette. Colorectal Dis 2021;23(1):326-7.

14. Yun Y, Miaojun W, Youzhen Z. Analysis of peripherally inserted central catheter-related infection factors in cancer patients undergoing chemotherapy and preventive strategies. Mod Clin Nurs 2010;9(8):10-2.

15. Yuan TH. Risk factors and nursing strategies of PICC catheterization complications in tumor chemotherapy patients. Mod Med Health 2019;35(9):1409-11.

16. Zhang KX, Tian $\mathrm{T}$. The effect of early nursing intervention in preventing mechanical phlebitis after PICC catheterization in tumor patients. China Rural Health 2018;134(8):49-50.

17. Kou H, Li Y. Analysis the effect of predictive nursing of on the indwelling time of skin puncture in patients with gastric cancer chemotherapy. Guide Chin Med 2018;16(9):276-7.

18. Zhang YY, Huang Y. Application effect of predictive nursing in prevention and treatment of complications of PICC catheterization in patients with cancer. China Mod Doct 2018;56(36):156-9.

This is an open access article distributed under the terms of the Creative Commons Attribution-NonCommercial-ShareAlike 3.0 License, which allows others to remix, tweak, and build upon the work non-commercially, as long as the author is credited and the new creations are licensed under the identical terms

This article was originally published in a special issue,
"Therapeutic Perspectives in Biomedical Research and Pharma-
ceutical Sciences and their Nursing Methods"
Indian J Pharm Sci 2021:83(4)Spl issue "173-177"

